\title{
Referees for Volume 1(1-2) of the Pesquisa e Ensino em Ciências Exatas e da Natureza (2017)
}

The Editorial Board of Pesquisa e Ensino em Ciências Exatas e da Natureza would like to thank the referees listed below for their critical review of articles submitted for Volume 1(1-2) (2017).

- M. Sc. Airan dos Santos Protázio.

IFBA-Irecê/BA/Brazil

- Dr. André Lincoln Barroso de Magalhães. UFSJ-Ouro Branco/MG/Brazil

- M. Sc. Adriano Medeiros de Souza.

- Dr. Afonso Henrique S. Maia Leal Gantus Francisco. ..UFPB/CGEN/DSE-João Pessoa/PB/Brazil

- M. Sc. Bruno Eleres Soares. .ICMBio/MMA-João Pessoa/PB/Brazil

- Dr. Cynthia Lara de Castro Manso. UFRJ/IB/DE-Rio de Janeiro/RJ/Brazil

- Dr. Daniela Cristina de Oliveira Rosa UFS/DB- Itabaiana/SE/Brazil

- M. Sc. Dimítri de Araújo Costa. UFRJ/IB/DE-Rio de Janeiro/RJ/Brazil

- M. Sc. Ednei de Almeida Mercês. /PRODEMA-João Pessoa/PB/Brazil

- Dr. Francis Luiz Santos Caldas.

- Dr. Gumercindo Souza Lima.. UEFS-Feira de Santana/BA/Brazil

- Dr. José Anderson Feijó da Silva. ..FISE-Aracaju/SE/Brazil

- Dr. José Eriberto de Assis

- M. Sc. Luiz Paulo Araújo da Silva.

- Dr. Marcelo Aloisio Sulzbacher. UFV/CCA/DEF-Viçosa/MG/Brazil

- M. Sc. Marília Pessoa Silva. ...UFPB/CCEN/DSE-João Pessoa/PB/Brazil ...UFPB/CCEN/DSE-João Pessoa/PB/Brazil ..UFSM-Santa Maria/RS/Brazil

- M. Sc. Maristela de Lima Bueno. ..USP/IB-São Paulo/SP/Brazil

- Dr. Mauricio Martins da Rocha.

- Dr. Mauro Luís Triques.

- Dr. Rafael Prezzi Indicatti. .UNESPAR/FAFIPAR-Paranaguá/PR/Brazil
.....................USPMG/MZSP-São Paulo/SP/Brazil

- Dr. Raone Beltrão Mendes .UNESPAR/FAFIPAR-Paranaguá/PR/Brazil
......................USPM/MZSPP-ICB/DZ-Belo Horizonte/MG/Brazil

- Dr. Renata Bartolette de Araujo

- Dr. Ricardo José de Carvalho Paiva.

- Dr. Ricardo Rodrigues dos Santos.

- Dr. Rodrigo Brincalepe Salvador.

- Dr. Samuel Cardozo Ribeiro. ...Instituto Butantan-São Paulo/SP/Brazil ..UFS/CCBS/DE-São Cristóvão/SE/Brazil .UFS/CCBS/DBI-São Cristóvão/SE/Brazil ...UFPE/CTG/DOCEAN-Recife/PE/Brazil ...UFMA/CCAA-Chapadinha/MA/Brazil

- Dr. Telton Pedro Anselmo Ramos. ..EKUT/Germany

- Dr. Tiago Fernandes Carrijo. UFCA/IFE-Brejo Santo/CE/Brazil

- Dr. Vanderlei Secretti Decian UFPB/CGEN/DSE-João Pessoa/PB/Brazil

- Dr. Waltécio de Oliveira Almeida. URI/CT-Erechim/RS/Brazil

- Dr. Walter Ramos Pinto Cerqueira URCA/DQB-Crato/CE/Brazil

- Dr. Yzila Liziane Farias Maia de Araújo

- M. Sc. Willian Fabiano da Silva. ..UEFS/DCB-Feira de Santana/BA/Brazil UFS/CCBS/DBI-São Cristóvão/SE/Brazil ..UFPB/CCEN/DSE-João Pessoa/PB/Brazil 


\section{EDITORIAL TEAM}

\section{EDITORS-IN-GHIEF}

Dr. Carlos Davidson Pinheiro;

Dr. Eudes Leite de Lima;

Dr. Heydson Henrique Brito da Silva;

Dr. Silvio Felipe Barbosa de Lima;

\section{EDITORIAL AND SCIENTIFIC BOARD}

Dra. Albaneide Fernandes Wanderley;

Dr. Carlos Davidson Pinheiro;

M. Sc. Edilson Leite da Silva;

M. Sc. Eudes Leite de Lima;

Dr. Everton Vieira da Silva;

Dr. Ezequiel Fragoso Vieira Leitão;

Dr. Fernando Antônio Portela da Cunha;

M. Sc. Francisco Carlos Pinheiro da Costa;

M. Sc. Gustavo de Alencar Figueiredo;

Dr. Heydson Henrique Brito da Silva;

Dr. João Maria da Silva;

Dr. José Deomar de Souza Barros;

Dra. Letícia Carvalho Benitez;

Dra. Mirleide Dantas Lopes;

Dr. Paulo Roberto de Medeiros;

Esp. Rosana Ferreira de Alencar;

Dr. Silvio Felipe Barbosa de Lima;

\section{SECTION SCIENTIFIC EDITORS}

Dra. Albaneide F. Wanderley (Chemistry); Dr. Carlos Davidson Pinheiro (Chemistry); Dr. Everton Vieira da Silva (Chemistry); Dr. Ezequiel Fragoso Vieira Leitão (Chemistry); Dr. Fernando A. Portela da Cunha (Chemistry); Dr. Luciano Leal de Morais Sales (Chemistry); M. Sc. Edilson Leite Silva (Computer Science); M. Sc. Eudes Leite de Lima (Mathematics); M. Sc. Franciélia L. de Sousa (Mathematics); Dr. Douglas Fregolente (Physics);

M. Sc. Gustavo de Alencar Figueiredo (Physics); Dr. Heydson Henrique Brito da Silva (Physics); Dra. Mirleide Dantas Lopes (Physics);

M. Sc. Francisco Carlos P. Costa (Biology); M. Sc. Hugo da Silva Florentino (Biology); Dra. Letícia Carvalho Benitez (Biology); Dr. Paulo Roberto de Medeiros (Biology); Esp. Rosana Ferreira Alencar (Biology); Dr. Silvio Felipe Barbosa de Lima (Biology); Dr. Udson Santos (Biology);

\section{ASSOCIATE EDITORS}

Dr. Alessandre Pereira Colavite (UFPB/DSE); Dr. Alexandre Campos (UFCG/CCT/UAF);
Dr. Angel Ramon S. Delgado (UFRRJ/ICE/DM); Dra. Antonia Arisdélia A. Feitosa (UFPB/DSE); M. Sc. Arielson Santos Protázio (UFRB/CCAAB); Dr. Carlos Alexandre Borges Garcia (UFS/DQI); Dr. Carlos A. R. Vera-Tudela (UFRRJ/ICE/DM); Dra. Claudia Mazza Dias (UFRRJ/IM); Dra. Daniela Franco Carvalho (UFU/ICB/IB); Dra. Elisabete Maria Zanin (URI/FURI); Dr. Etielle Barroso de Andrade (IFPI); Dra. Eugenia Jacira Bolacel Braga (UFPel/IB); Dr. Felipe Wartchow (UFPB/DSE);

Dr. Gilberto Gonçalves Rodrigues (UFPE/CCB); Dr. Gustavo Benitez Alvarez (UFF/CT/EEIMVR); Dr. Joaquim Evêncio Neto (UFRPE/DMFA); Dr. José Marcos de Castro Nunes (UFBA/IB/DB); Dr. Jose Ricardo Miras Mermudes (UFRJ/IB); Dr. Leonardo Esteves Lopes (UFV/CEDAF); Dra. Lydia Dayanne Maia Pantoja (UECE/CCS); Dr. Manoel dos Santos Filho (UNEMAT/DBio); Dr. Marcelo Fulgêncio Guedes Brito (UFS/DB); Dr. Marcelo V. Fukuda (UFJF/ICB/DZOO); Dr. Marcio Bernardino da Silva (UFPB/DSE); Dra. Marla Ibrahim U. de Oliveira (UFS/DBI); Dr. Martin Lindsey Christoffersen (UFPB/DSE); Dr. Mauro de Melo Júnior (UFRPE/DB); Dra. Michela Borges (UNICAMP/IB/MZ); Dr. Pablo Ariel Martinez (UFS/CCBS/DBI); Dr. Patrício Borges Maracajá (UFCG/CCTA); Dr. Paulo Henrique R. Peixoto (UFPE/CAA); M. Sc. Raul Azevedo (UFCA/CCAB); Dra. Renata Perpetuo Reis (IPJBRJ); Dra. Rita de Cássia M. C. Rodriguez (UFPel/IB); Dr. Rodrigo Giesta Figueiredo (UFES/CCENS); Dra. Rosane F. de Oliveira (UFRRJ/ICE/DM); Dr. Samuel Vieira Brito (UFMA/CCAA); Dr. Saulo Pomponet Oliveira (UFPR/DMAT); Dr. Sérgio André Fontes Azevedo (UFPB/DF); Dr. Silvânio Silvério L. da Costa (UFS/NUPEG) Dra. Thatyara Freire de Souza (UFERSA/CMPF); Dr. Wagner de S. Tassinari (UFRRJ/ICE/DM); Dr. Wedson de Medeiros S. Souto (UFPI/CCN);

\section{ASSISTANT AND PRODUCTION EDITORS}

Sr. José Mateus Sarmento (UFCG); Sr. Emanuel Evaristo de Sousa (UFCG); Sr. Gustavo Tavares de Abreu (UFCG); Dr. Onireves Monteiro de Castro (UFCG);

\section{OPERATIONAL TECHNICAL SUPPORT AND} GRAPHIC DESIGNER

Sr. Antonio Lourenço de Araújo Filho; Sr. Delio Jackson Dantas Duarte; 